\title{
The link between attention-deficit hyperactivity disorder symptoms and entrepreneurial orientation in Japanese business owners
}

\author{
Annelot Wismans ${ }^{1,2}$ (D) $\cdot$ Katsuyuki Kamei ${ }^{4} \cdot$ Roy Thurik $^{1,2,3} \cdot$ Olivier Torrès $^{3,5}$
}

Received: 26 August 2020 / Accepted: 10 October 2020 / Published online: 29 October 2020

(c) The Author(s) 2020

\begin{abstract}
Several studies have observed a relationship between (subclinical levels of) attention-deficit hyperactivity disorder (ADHD) and entrepreneurship. Recently, Yu et al. (Entrep Theory Pract https://doi.org/10.1177/1042258719892987, 2019) and Wismans et al. (Appl Psychol 69:1093-1112 https://doi.org/10.1111/apps.12247, 2020) observed a positive association between hyperactivity and entrepreneurial orientation (EO). The present study seeks to replicate these findings in an entirely different culture, that of Japan. Moreover, because of the low internal consistency of the hyperactivity measure across studies, we study the individual contributions of the hyperactivity items. Using a sample of 255 Japanese business owners, we conducted OLS regressions and PLS-structural equation modelling. The results of our study on the ADHD-EO relationship in Japan are partly in line with previous findings: the positive link between hyperactivity and EO is again confirmed. In contrast with the results of previous studies, we find a positive link between overall ADHD symptoms and EO using both methods and a positive link between attention-deficit and EO using one of the methods (PLS-SEM). Cultural differences may explain these different findings. When the two hyperactivity items are separated, they appear to have different associations with EO. One item, measuring mental hyperactivity, is strongly positively associated with EO, whereas the other item, measuring physical hyperactivity, is not associated with EO. This result is a new and interesting finding that is replicated in the current study using the French dataset of Wismans et al. (Appl Psychol 69:1093-1112 https://doi.org/10.1111/apps.12247, 2020) but which warrants further research.
\end{abstract}

Keywords Attention-deficit · Hyperactivity - ADHD · Entrepreneurial orientation · Japan · Replication

JEL Classification J24 · L26

Annelot Wismans

wismans@ese.eur.nl

Extended author information available on the last page of the article 


\section{Introduction}

The positive psychology movement has encouraged the study of human thoughts, feelings and behaviours with a focus on strengths instead of deficits-for example, using the concept of psychological capital (Csikszentmihalyi and Seligman 2000; Nolzen 2018). Related to this, a stream of research emerged that advocated a change from the deficit-focused view of mental disorders to one that is oriented towards the benefits that may result from the traits associated with these disorders (Lesch 2018; Jamison 2005; Galvez et al. 2011). In the field of entrepreneurship, several studies have been conducted that link symptoms of mental disorders, specifically attention-deficit hyperactivity disorder (ADHD), to entrepreneurship (Wiklund et al. 2018; Verheul et al. 2015; Antshel 2018; Hatak et al. 2021). ADHD is a neurodevelopmental disorder that is characterized by symptoms of inattention and/or hyperactivity. Often, the disorder arises during childhood and persists into adulthood (American Psychiatric Association 2013). The clinical diagnosis of ADHD has been associated with occupational impairments, such as lower educational attainment, lower work performance, lower supervisor ratings and greater probability of being fired (Antshel 2018).

Recently, studies have reported a positive link between ADHD and different facets of entrepreneurship, such as entrepreneurial intention, the choice to become self-employed and entrepreneurial orientation. A number of these studies have taken a clinical perspective by studying the diagnosis of ADHD (Dimic and Orlov 2014; Lerner et al.2019), whereas others have taken a subclinical perspective by studying the presence of ADHD symptoms (Verheul et al. 2015, 2016; Canits et al. 2019). For a review of this literature, see Antshel (2018).

To date, two studies have investigated the relationship between ADHD symptoms and entrepreneurial orientation (EO): Yu et al. (2019) and Wismans et al. (2020). EO is the strategic orientation of a firm. The central premise of EO is that a firm can be more (or less) entrepreneurial, and it refers to how entrepreneurial activities are performed. EO has three subdomains: risk-taking, innovativeness and proactiveness (Miller 1983). EO can also be measured at the individual level (Covin and Slevin 1988). In their paper, Wismans et al. (2020) studied the overall link between weighted ADHD symptoms and individual-level EO and found no relationship on this level. Both Yu et al. (2019) and Wismans et al. (2020) studied the effects of the ADHD symptoms attention-deficit and hyperactivity individually and found hyperactivity to be positively linked to EO. Disentangling the ADHD-EO relationship even further, Wismans et al. (2020) distinguished between the subdimensions of EO. They showed that the hyperactivity-EO link was driven primarily by the risk-taking and proactiveness dimensions of EO.

$\mathrm{Yu}$ et al. (2019) used one sample of Spanish entrepreneurs and one made up of mostly American entrepreneurs, whereas Wismans et al. (2020) used a French sample. The current study aims to replicate the findings of Yu et al. and Wismans et al. in an entirely different culture, i.e., the Japanese culture. We follow the approach of Wismans et al. (2020) by both studying the overall link between ADHD and EO and by differentiating between the subdimensions of both 
constructs. Moreover, across studies, the widely used measure of ADHD symptoms-the ASRS-6- has shown low internal consistency, especially for hyperactivity symptoms. In our study, similarly, we found a low internal consistency for the hyperactivity measure. Therefore, supported by the notion of Wismans et al. (2020) that the two items making up the hyperactivity measure could possibly capture distinct effects, we analyse whether these indeed have different relationships with entrepreneurship. Hence, we perform an additional analysis in which we distinguish between different types of hyperactivity, which are labelled mental and physical hyperactivity. We perform our analyses using both ordinary least squares (OLS) and partial least squares-SEM (PLS-SEM) to follow the approach of the studies we replicate.

Cultural values and norms will either converge or conflict with a country's ability to develop a strong EO. Traditionally, harmony, cooperation, status quo and consensus are values that characterize Japanese culture. This results in a lower risk-taking tendency, where people search for and prefer safe jobs (Lee and Peterson 2000). Moreover, in Japan, high scores on Hofstede's power distance, uncertainty avoidance and masculinity indices and a low score on individualism contribute to lower rates of entrepreneurial activity, risk-taking and proactiveness (Okamuro et al. 2017; Kreiser et al. 2010). Relative to other advanced countries, entrepreneurial attitudes and entrepreneurial activity are considerably lower in Japan (Bosma et al. 2020). Studies show that Japanese entrepreneurs are more society-oriented, meaning that they are in search of social recognition, compared with Silicon Valley entrepreneurs, who are motivated more by individualistic factors. Family members' opinions are very influential in becoming entrepreneur in Japan (Suzuki et al. 2002). Finally, the gender gap in Japan is relatively large (Bosma et al. 2020), which is also visible in our sample (only $7.83 \%$ females).

We find that hyperactivity is also positively linked to EO in Japanese small business owners, which is in line with the findings of previous studies. However, the association is driven by other subdimensions of EO than found in the study by Wismans et al. (2020). We also have some new and surprising findings. Using one of the methods, PLS-SEM, we find a positive association between attention-deficit and EO. Moreover, we identify a positive relationship between the higher-level constructs of ADHD and EO. Finally, the additional analysis of the hyperactivity items shows that the two items are related to EO differently. One of the items, capturing 'mental hyperactivity', is positively related to EO, whereas the other item, capturing 'physical hyperactivity', is not related to EO.

Replication studies are important to improve the base of empirical evidence. By conducting this study, we both add to the generalizability of previous findings and extend those findings by including an additional analysis (Block and Kuckertz 2018). The importance of the present paper is therefore threefold. First, as others have pointed out, studying the positive implications of ADHD can contribute to a more positive view of this mental disorder. Entrepreneurship could be an occupation in which ADHD-(type behaviour) proves to be an asset instead of a liability, though further research is necessary that studies the clinical diagnosis. Moreover, the link between ADHD and entrepreneurial success should be studied. Second, by replicating previous results, we add to the robustness of the hyperactivity-EO relationship 
by replicating it in an entirely different culture. Finally, by assessing the hyperactivity items separately, we help to elucidate possible causes of the low internal consistency of the hyperactivity construct. This leads to a novel finding that different types of hyperactivity, viz. mental and physical hyperactivity, are differently associated with EO. We go one step further by replicating this new finding using the dataset of Wismans et al. (2020).

Taken together, we replicate findings from original French, Spanish and predominantly American data sets, find additional and new results using a new Japanese data set and replicate them again using the original French data set.

We will refrain from an extensive literature review. Since our first aim is to replicate previous studies, we do not justify hypotheses and rather refer to previous work for substantiation (Antshel 2018; Yu et al. 2019; Wismans et al. 2020). Moreover, the additional analysis of the hyperactivity items led to a surprise finding that was not based on a hypothesis. This finding generates a hypothesis in need of scientific justification to which we will return in the conclusion. The paper is therefore structured as follows. We will start with the methods section, in which the dataset, measures and methodology are described. Next, the results are discussed. Finally, the paper ends with a discussion and the conclusion of our findings.

\section{Methods}

\subsection{Dataset}

The data were collected by Amarok Japan. Amarok Japan was founded in September 2010 as the Japanese branch of the French Amarok research organization. Amarok studies small business owners' beliefs, attitudes and behaviours in relation to their mental and physical health. Primary data were collected by a research team of the project 'Amarok Keieisha Kenko Anshin Action' (Amarok Manager Health Security Action) in Japan between March and October 2017. This project organized by Amarok Japan is a joint effort of Kansai University, Anshin Zaidan and Otsuma Women's University. Anshin Zaidan is an insurance company specializing in casualty insurance for SMEs. Only those entities that are SMEs according to the Japanese definition (Japan SME Basic Act, Article 2, 1999) can be clients for Anshin Zaidan's insurance activities.

Some 10,000 postal invitations were sent to the clients of Anshin Zaidan. In addition, employees of Anshin Zaidan directly approached their 3000 SME customers. Furthermore, an invitation was put in the bulletin of Anshin Zaidan. After agreeing to participate, entrepreneurs were interviewed by telephone. Participants were excluded based on the same rules applied by Wismans et al. (2020): entrepreneurs with a firm size larger than 250 employees or who owned less than $5 \%$ of the financial capital of their firm were excluded. Moreover, participants with missing values on ADHD or EO measures were excluded too. This resulted in a dataset consisting of 255 Japanese small business owners.

The average age of the entrepreneurs was 61.83 years, with a standard deviation (SD) of 11.34 years, and $7.83 \%$ are female. Regarding education, $50.61 \%$ of the 
interviewees had completed at least a two-year degree after high school. Participating entrepreneurs had 17.59 employees on average with a SD of 24.71.

\subsection{Measures}

\subsubsection{ADHD}

ADHD was measured by the Adult ADHD Self-Report Scale v1.1 Screener (ASRS6), created by the World Health Organization (Kessler et al. 2005). This questionnaire is the short version of the ASRS-18 and consists of the 6 most predictive items from the full 18-item measure. The ASRS-6 can be used as a starting point to recognize the symptoms and signs of ADHD. Previous studies have used this screener to study subclinical levels of ADHD. Factor analysis has shown that it can be used to study the subdimensions of ADHD, attention-deficit and hyperactivity (Hesse 2013). For all items, participants indicate how they have felt and conducted themselves over the past 6 months on a scale of 1 ('Never') to 5 ('Very Often'). To assess overall ADHD symptoms, a weighted average of the items related to hyperactivity (50\%) and attention-deficit (50\%) was created.

Four items are related to attention-deficit symptoms. An example of an item is, 'How often do you have trouble wrapping up the final details of a project, once the challenging parts have been done?'

Two items are related to hyperactivity symptoms: 'How often do you fidget or squirm with your hands or feet when you have to sit down for a long time?' (Hy1) and 'How often do you feel overly active and compelled to do things, like you were driven by a motor?' (Hy2).

Table 1 shows the Cronbach's alphas, means and correlations of all variables. Both attention- deficit $(\alpha=.64)$ and hyperactivity $(\alpha=.32)$ have low internal consistency. Previous studies have likewise reported the same issues (Wismans et al. 2020; Yu et al. 2019; Kessler et al. 2007), although the internal consistency level of hyperactivity in our Japanese sample is notably extreme. An explanation is that very high alphas would not be expected, since to create the ASRS-6, the least redundant items of the ASRS-18 were selected (Kessler et al. 2007). However, to follow up on the suggestion of Wismans et al. that the two items possibly capture distinct parts of hyperactivity, we study their separate effects. Looking at the items of hyperactivity, it seems that Hyl is related to a more physical type of hyperactivity (fidgeting/squirming with hands or feet), whereas Hy1 is related to a more mental type of hyperactivity ('being driven by a motor').

\subsubsection{EO}

EO was measured at the individual level using the scale of Covin and Slevin (1988). This scale consists of 9 items. Participants had to state how much they agree with each item by considering the last three years of their lives on a 7-point Likert scale (1: strongly disagree; 7: strongly agree). Each of the subdimensions of innovativeness, proactiveness and risk-taking is measured by 3 items. An example of an item 
measuring innovativeness is, 'You have largely renewed your range of products or services'. One of the items capturing proactiveness is as follows: 'You typically tend to respond to the actions of your competitors, rather than precede them' (reversed). Finally, risk-taking was amongst others assessed by, 'You tend to favour high-risk projects'. To assess total EO, the average of the 9 items was used.

\subsubsection{Control variables}

We used the exact same control variables as those used by Wismans et al. (2020). These were age, gender (female $=1$ ), education level, firm size (dichotomized: $0: \leq 10$ employees; $1:>10$ employees) and mental and physical hyperactivity. Mental and physical hyperactivity were both measured by 1 item asking to describe one's mental or physical health over the last months on a 5-point Likert scale (1: Very bad, 5: Excellent). Since Amarok Japan is set up as a branch of Amarok France, education level was measured in an ordinal manner according to the French educationsystem (1: no secondary education; 2 : vocational education, not high school; 3 : high school; 4 : high school $+2 / 3$ years of further education; 5 : high school $+4 / 5$ years of further education; 6: $\mathrm{PhD}$ ).

\subsection{Methodology}

Since we try to replicate previous findings, we will use the methods applied by Wismans et al. (2020). We use ordinary least squares (OLS) and partial least squares structural equation modelling (PLS-SEM) to study the relationship between ADHD and EO and their subdimensions. PLS-SEM is a path modelling technique that is suitable for the nature of our constructs ADHD and EO. Both ADHD and EO are constructed as first-order reflective, second-order formative measures in PLS-SEM. For reasons of parsimony, PLS-SEM is used only for the highest measurement level of EO and not for the analyses of its subdimensions.

\section{Results}

The results of the analyses can be found in Table 2 .

\subsection{ADHD and EO}

Estimating the relationship using OLS regressions, weighted ADHD is positively associated with EO $(\beta=.16, p<.01)$. Using PLS-SEM, we find a similar positive relationship between the two constructs $(\beta=.20, p<.01)$. Looking at the subdimensions, ADHD is positively related to innovativeness at a $10 \%$ significance level $(\beta=.11, p<.10)$, not related to proactiveness and significantly related to risk-taking $(\beta=.26, p<.01)$. 


\subsection{Attention-deficit and hyperactivity and EO}

Looking at the subdimensions of ADHD using OLS, we find no link between attention-deficit and total EO, and we find a positive association between hyperactivity and total EO that is significant at the $10 \%$ level $(\beta=.11, p<.10)$. Using PLSSEM, we find that both attention-deficit $(\beta=.16, p<.05)$ and hyperactivity $(\beta=.15$, $p<.05)$ are positively and significantly related to total EO.

In terms of the EO subdimensions, we find that attention-deficit is not related to innovativeness, while hyperactivity is associated with innovativeness only at the $10 \%$ significance level $(\beta=.12, p<.10)$. Both attention-deficit and hyperactivity are not related to proactiveness, but both show a significant positive relationship with risk-taking (attention-deficit: $\beta=.22, p<.01$; hyperactivity: $\beta=.13, p<.05$ ).

\subsection{Mental and physical hyperactivity and EO}

Since the internal consistency of the hyperactivity construct is low, we conducted OLS and PLS-SEM analyses distinguishing between the two items of hyperactivity, where the first item seems to capture physical hyperactivity (Hy1), and the second seems to capture mental hyperactivity (Hy2). The results can be found in Table 2.

We find that physical hyperactivity (Hy1) is not associated with EO or its subdimensions, when analysing the link using both OLS and PLS-SEM. However, mental hyperactivity (Hy2) is positively related to EO estimated both by OLS and PLS-SEM (OLS: $\beta=.16, p<.05$; PLS-SEM: $\beta=.15, p<.05$ ). Looking at the subdimensions of EO, mental hyperactivity is associated with innovativeness $(\beta=.14$, $p<.05$ ), not with proactiveness, and only at the $10 \%$ significance level with risktaking $(\beta=.12, p<.10)$.

\subsubsection{Replication in dataset of Wismans et al. (2020)}

Because of the strong difference that we find in the relationship of the two hyperactivity items with EO, we studied whether this distinction was also present in the dataset that was used by Wismans et al. (2020) in their study. The results of this replication can be found in Table 3. In their dataset, consisting of 788 French SME owners, physical hyperactivity (Hy1) is likewise not related to EO or its subdimensions. As in our Japanese sample, we find that mental hyperactivity (Hy2) is strongly related to EO estimated both by OLS and PLS-SEM (OLS: $\beta=.13, p<.01$; PLSSEM: $\beta=.12, p<.01)$ in their dataset as well. However, the effect on the subdimensions is different: mental hyperactivity (Hy2) is not associated with innovativeness, associated with proactiveness $(\beta=.13, p<.01)$ and associated with risk-taking $(\beta=.11, p<.01)$. 


\section{Discussion and conclusions}

The primary aim of the present study was to replicate the findings of previous studies by $\mathrm{Yu}$ et al. (2019) and Wismans et al. (2020) in an entirely different culture. Both studies document a positive link between hyperactivity and EO and no link between attention-deficit and EO. Furthermore, Wismans et al. (2020) also studied the higher-level relationship and found no link between total ADHD and EO. In the present study, we analysed a dataset of Japanese small business owners.

In contrast with the results of Wismans et al. (2020), the present study found an association between the higher-level constructs of ADHD and EO. ADHD was found to be positively related to EO, which appears to be primarily driven by a link with the risk-taking dimension and partly by a weak link with the innovativeness dimension. Wismans et al. (2020) did not find a link between ADHD and EO, although they did find a link between ADHD and the risk-taking subdimension, for which we find a strong link, as well.

Similar to previous studies, we find a positive link between hyperactivity and EO. When distinguishing between the subdimensions of EO, we find that the hyperactivity-EO link is driven by different subdimensions than in the study of Wismans et al. (2020). In their sample, the relationship was driven mainly by the proactiveness and risk-taking subdimensions of EO. In contrast, in our Japanese sample, the hyperactivity-EO relationship is mainly driven by risk-taking and, to a smaller extent, by innovativeness and not by proactiveness.

Contrary to previous studies, we find attention-deficit to be positively related to EO when applying PLS-SEM, though the link is not significant when studied by using OLS. This positive link was not found in the previous two studies conducted (Yu et al. 2019; Wismans et al. 2020). Wismans et al. (2020) even found a negative link between attention-deficit and proactiveness. In our Japanese sample, we find that attention-deficit is strongly linked to the risk-taking dimension and not to the other subdimensions of EO. It thus seems that the positive ADHD-EO link in our Japanese sample of business owners is driven by positive relations of both attentiondeficit and hyperactivity with EO.

The fact that we find a positive association between ADHD/attention-deficit and EO in our sample that was not found before could be due to cultural differences between the datasets used in previous studies and the dataset we use. As discussed in the introduction, studies show that national culture, by way of both values and institutions, impacts the EO in a country (Kreiser et al. 2010). Japanese culture, characterized by high power distance, uncertainty avoidance, masculinity and collectivism, contributes to lower rates of entrepreneurial activity, risk-taking and proactiveness (Lee and Peterson 2000; Okamuro et al. 2017; Kreiser et al. 2010). Second, although ADHD is found to be a cross-cultural valid disorder and no differences in prevalence rates have been documented to date (Davis et al. 2012; Polanczyk et al. 2007), it is likely that culture affects behaviour in subclinical samples. In Japanese culture, substantial emphasis is placed on harmonious behaviour and 
interpersonal sensitivity, which could cause people to underreport behaviour that is typical for ADHD (Davis et al. 2012). This is in line with the lower average values of both hyperactivity and attention-deficit on the ASRS-6 that we find in our sample compared with the French sample of Wismans et al. (2020). Both these aspects could affect the strength and direction of the relationships that we find in our study. However, more research is needed to explain the dynamics that underlie the positive link that is found between ADHD/attention-deficit and EO in our Japanese sample that was not found before.

The second aim of the present study was to obtain a better understanding of the low reliability of the hyperactivity construct. Since several studies showed that the hyperactivity measure has low internal consistency, Wismans et al. (2020) noted that the two items making up the construct could capture distinct effects. By analysing the two hyperactivity items separately, we found that one of the items is not linked to EO (Hy1), whereas the other item has a strong positive link with EO (Hy2). The item not related to EO seems to measure a more physical type of hyperactivity, whereas the other items seem to measure a more mental type of hyperactivity. Additionally, to check for the robustness of this finding, we turned to the dataset of Wismans et al. (2020). In their dataset of French SME owners, the same difference in the associations of the mental and physical hyperactivity items with EO was present. The fact that we also replicate it in another sample increases our belief in the robustness of this finding.

ADHD in adults is usually characterized by mental restlessness instead of physical hyperactivity, which is more common in children. As children with ADHD grow older, overt hyperactivity symptoms often evolve into more internal restlessness during adolescence and adulthood (American Psychiatric Association 2013; Ingram et al. 1999). Therefore, the strong result for the mental hyperactivity item could be caused by the fact that mental hyperactivity more accurately captures ADHD symptoms that are present in adults. However, for a precise interpretation, more research is needed on whether the two items indeed relate to these two expressions of hyperactivity. Furthermore, future results would increase in reliability if composite measures of 'physical' and 'mental' hyperactivity comprising of multiple items would be constructed and used. An interesting avenue is to use the Internal Restlessness Scale (Iwaszuk et al. 1997; Weyandt et al. 2003), which was designed to capture mental restlessness in adults with ADHD. In conclusion, we present a novel and interesting result, though further research is necessary.

We consistently obtain stronger results (lower errors) when using PLS-SEM compared with using OLS, which could be explained by the fact that the methods make different statistical assumptions but also by the difference in how the constructs are 'built' in the two methods. Within our OLS model, we used the average value of the items of the scales (or weighted average in case of ADHD), which means that every item or symptom has an equal weight. In contrast, in PLS-SEM, more weight is given to items with higher predictive validity. 
This study makes several contributions. First, the fact that we replicate the hyperactivity-EO association in a totally different cultural context adds to the robustness of this link. Second, by studying the hyperactivity items separately, we have provided insights into the associations of different types of hyperactivity. For further research, it is important to study whether we can indeed state that this is a distinction between physical and mental hyperactivity or whether the items capture something else. Third, the fact that we replicate the positive relationship between ADHD symptoms and entrepreneurship could improve and de-stigmatize the view of ADHD. It is even more encouraging for the implications of ADHD symptoms in the Japanese population that we also find a positive link between attention-deficit and EO. However, subclinical levels of ADHD were studied, so the results will not necessarily hold for those with a full-blown clinical diagnosis of ADHD. Furthermore, the present research does not tell us whether the positive relationship between ADHD symptoms and EO also translates to entrepreneurial success. However, the positive relationship with firm performance through EO was established in the study by $\mathrm{Yu}$ et al. (2019). Another promising avenue for future research is the study of the relationship of other mental disorders-for example, dyslexia, which is often related to ADHD-to entrepreneurship.

A limitation of the present study is that we have used self-reported data measured at only one moment in time. Future research should make use of different types of observations involving reports by clinical psychologists and controlling for consistency over time. Furthermore, we found differences between the results of our study and those of previous studies. Here, we expect that culture could play a role, though with the information we have of only a limited number of societal settings, we are unable to draw any conclusions. Future research should investigate whether and to what degree cultural differences underlie the differences between our findings and those of previous studies.

The robust link between ADHD symptoms and EO could have positive consequences in two directions. First, for people struggling with these symptomsmostly associated with detrimental outcomes in employment—starting a business could provide a suitable occupation. Yu et al. (2019) showed that ADHD symptoms are related to firm performance through EO. Second, these new businesses would lead to less unemployment and will have a positive effect on regional development and economic performance (Neumann 2020). To stimulate entrepreneurship among people with ADHD symptoms, an interesting avenue for further research could be to study the effect of entrepreneurship education on adolescents with and without ADHD symptoms. As stated by Brüne and Lutz (2020), 
scholars should differentiate between different groups when studying the effects of entrepreneurship education programmes. Moreover, it would be interesting to study the influence of role models, for example, successful entrepreneurs diagnosed with ADHD, on students with ADHD symptoms. Studies show that role models have positive effects on entrepreneurial intentions, especially when the perceived similarity is high (Abbasianchavari and Moritz 2020).

In conclusion, in the current study, we observe a positive association between ADHD and EO. Additionally, we replicate the positive relationship found between hyperactivity symptoms and EO found by Yu et al. (2019), Wismans et al. (2020) in an entirely different culture. Moreover, using PLS-SEM, we find a positive relationship between attention-deficit symptoms and EO that was not found previously. Finally, we show that the items underlying the hyperactivity construct are related to EO differently. It appe ars that a more mental type of hyperactivity is positively related to EO, whereas the more physical type of hyperactivity is not related to EO.

Acknowledgements The present research was supported by LabEx Entreprendre of the Universite de Montpellier (Montpellier Management, MOMA) which is funded by the French government (LabEx Entreprendre, ANR-10-Labex-11-01) as well as by the public research centre Montpellier Research in Management (EA 4557, Université de Montpellier). The present research was also supported by a grant of Erasmus University Rotterdam called Research Excellence Initiative (REI 2015). Finally, this research benefited from the support of the General Foundation Anshin Zaidan.

Open Access This article is licensed under a Creative Commons Attribution 4.0 International License, which permits use, sharing, adaptation, distribution and reproduction in any medium or format, as long as you give appropriate credit to the original author(s) and the source, provide a link to the Creative Commons licence, and indicate if changes were made. The images or other third party material in this article are included in the article's Creative Commons licence, unless indicated otherwise in a credit line to the material. If material is not included in the article's Creative Commons licence and your intended use is not permitted by statutory regulation or exceeds the permitted use, you will need to obtain permission directly from the copyright holder. To view a copy of this licence, visit http://creativecommons.org/licen ses/by/4.0/.

\section{Appendix}

See Tables 1, 2 and 3. 


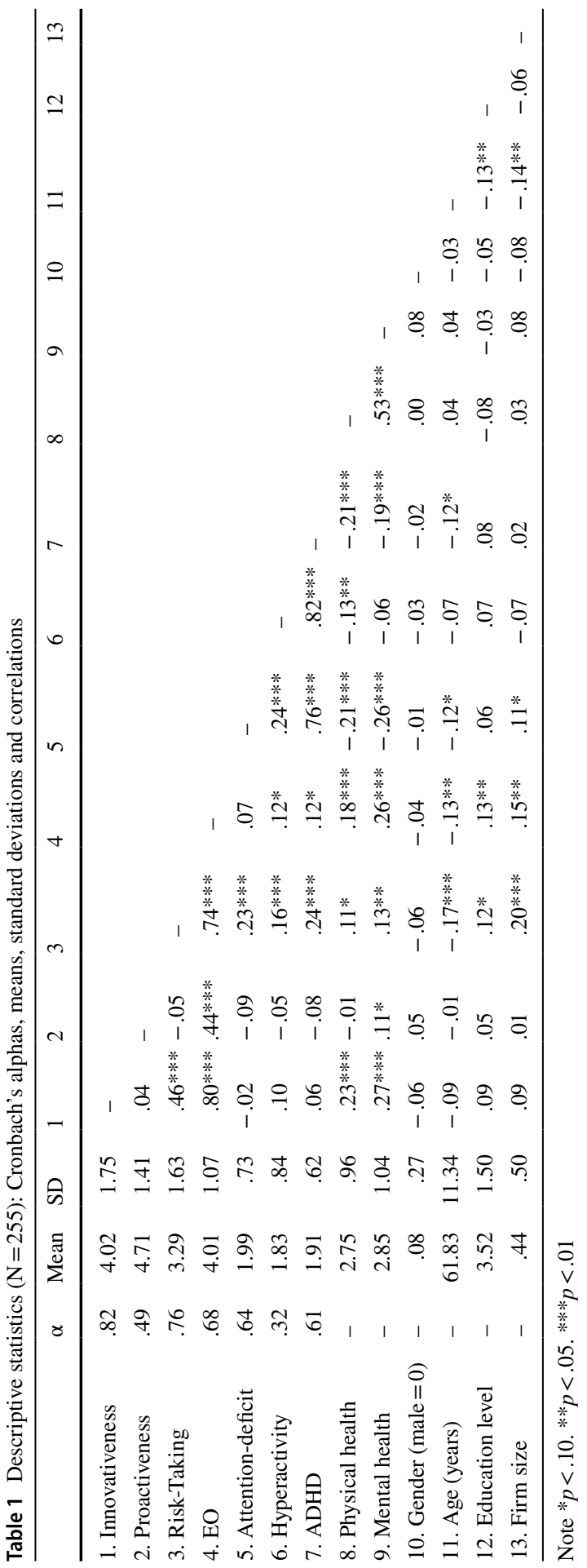


Table 2 Results from OLS regressions and PLS-SEM analysis $(\mathrm{N}=255)$

\begin{tabular}{|c|c|c|c|c|c|c|c|c|c|c|}
\hline & \multicolumn{2}{|c|}{$\begin{array}{l}\text { Innovative- } \\
\text { ness }\end{array}$} & \multicolumn{2}{|c|}{$\begin{array}{l}\text { Proactive- } \\
\text { ness }\end{array}$} & \multicolumn{2}{|c|}{ Risk-Taking } & \multicolumn{2}{|l|}{ Total EO } & \multicolumn{2}{|c|}{$\begin{array}{l}\text { Total EO } \\
\text { (PLS-SEM) }\end{array}$} \\
\hline & $\beta$ & $S E$ & $\beta$ & $S E$ & $\beta$ & $S E$ & $\beta$ & $S E$ & $\beta$ & $S E$ \\
\hline \multicolumn{11}{|l|}{$A D H D$ weighted total } \\
\hline ADHD & $.11^{*}$ & .17 & -.08 & .15 & \multicolumn{2}{|c|}{$.26 * * * .16$} & \multicolumn{2}{|c|}{$.16^{* * *} .10$} & \multicolumn{2}{|c|}{$.20 * * * .06$} \\
\hline Physical health & $.15^{* *}$ & .13 & -.10 & .11 & .11 & .12 & .10 & .08 & $.15^{* *}$ & .07 \\
\hline Mental health & $.21 * * *$ & .12 & $.15^{*}$ & .10 & $.12 *$ & .11 & $.24 * * *$ & $=07$ & $.21 * * *$ & .08 \\
\hline Gender $($ male $=0)$ & -.07 & .39 & .04 & .33 & -.04 & .36 & -.04 & .24 & -.07 & .06 \\
\hline Age (years) & -.07 & .01 & -.01 & .01 & $-.11 *$ & .01 & $-.10 *$ & .01 & $-.11 *$ & .06 \\
\hline Education level & .09 & .07 & .06 & .06 & $.11^{*}$ & .07 & $.13 * *$ & .04 & $.11^{*}$ & .06 \\
\hline Firm size & .05 & .21 & .01 & .18 & $.17 * * *$ & .20 & $.12 *$ & .13 & $.11 *$ & .06 \\
\hline$R^{2}$ & .12 & & .03 & & .16 & & .15 & & & \\
\hline \multicolumn{11}{|l|}{ Subdimensions ADHD } \\
\hline Attention-deficit & .02 & .15 & -.07 & .13 & $.22 * * *$ & .14 & .09 & .09 & $.16^{* *}$ & .06 \\
\hline Hyperactivity & $.12^{*}$ & .13 & -.04 & .11 & $.13 * *$ & .12 & $.11^{*}$ & .08 & $.15^{* *}$ & .06 \\
\hline Physical health & $.15^{* *}$ & .13 & -.10 & .11 & .11 & .12 & .10 & .08 & $.16^{* *}$ & .07 \\
\hline Mental health & $.20 * * *$ & .12 & $.14 *$ & .10 & $.13^{*}$ & .11 & $.24 * * *$ & .07 & $.20 * * *$ & .07 \\
\hline Gender $($ male $=0)$ & -.06 & .39 & .04 & .33 & -.05 & .36 & -.04 & .24 & -.07 & .06 \\
\hline Age (years) & -.07 & .01 & -.01 & .01 & $-.11 *$ & .01 & $-.10^{*}$ & .01 & $-.10^{*}$ & .06 \\
\hline Education level & .09 & .07 & .06 & .06 & $.11^{*}$ & .07 & $.13 * *$ & .04 & $.11 * *$ & .06 \\
\hline Firm size & .06 & .22 & .01 & .18 & $.15^{* *}$ & .20 & $.12 *$ & .13 & $.12 * *$ & .06 \\
\hline$R^{2}$ & .12 & & .03 & & .16 & & .15 & & & \\
\hline \multicolumn{11}{|l|}{ Hyperactivity differentiated } \\
\hline Attention-deficit & .00 & .156 & -.08 & .13 & $.21 * * *$ & .14 & .07 & .09 & $.16^{* *}$ & .06 \\
\hline Physical hyperactivity (Hy1) & .02 & .10 & -.10 & .08 & .04 & .09 & -.01 & .06 & .03 & .06 \\
\hline Mental hyperactivity (Hy2) & $.14 * *$ & .10 & .05 & .09 & $.12^{*}$ & .09 & $.16^{* *}$ & .06 & $.15^{* *}$ & .07 \\
\hline Physical health & $.15^{* *}$ & .13 & -.11 & .11 & .11 & .12 & .09 & .08 & $.16^{* *}$ & .07 \\
\hline Mental health & $.20 * * *$ & ${ }^{*} .12$ & $.14 *$ & .10 & $.13^{*}$ & .11 & $.24 * * *$ & .07 & $.20 * * *$ & .08 \\
\hline Gender $($ male $=0)$ & -.06 & .39 & .04 & .33 & -.05 & .36 & -.04 & .24 & -.07 & .05 \\
\hline Age (years) & -.08 & .01 & -.02 & .01 & $-.12 *$ & .01 & $-.11^{*}$ & .01 & $-.11 *$ & .06 \\
\hline Education level & .09 & .07 & .05 & .06 & $.10^{*}$ & .06 & $.12 * *$ & .04 & $.11^{*}$ & .06 \\
\hline Firm size & .07 & .22 & .02 & .19 & $.16^{* *}$ & .20 & $.13 * *$ & .13 & $.12 * *$ & .06 \\
\hline$R^{2}$ & .13 & & .04 & & .17 & & .16 & & & \\
\hline
\end{tabular}

Note $* p<.10, * * p<.05, * * * p<.01 . \beta=$ standardized beta 
Table 3 Additional analysis differentiating Hyperactivity items in dataset Wismans et al. (2020)

\begin{tabular}{|c|c|c|c|c|c|c|c|c|c|c|}
\hline & \multicolumn{2}{|c|}{$\begin{array}{l}\text { Innovative- } \\
\text { ness }\end{array}$} & \multicolumn{2}{|c|}{ Proactiveness } & \multicolumn{2}{|c|}{ Risk-Taking } & \multicolumn{2}{|c|}{ Total EO } & \multicolumn{2}{|c|}{$\begin{array}{l}\text { Total EO } \\
\text { (PLS-SEM) }\end{array}$} \\
\hline & $\beta$ & $S E$ & $\beta$ & $S E$ & $\beta$ & $S E$ & $\beta$ & $S E$ & $\beta$ & $S E$ \\
\hline Attention-deficit & -.02 & .08 & $-.11 * * *$ & .07 & -.01 & .07 & -.06 & .05 & $-.06^{*}$ & .04 \\
\hline Physical hyperactivity (Hy1) & -.02 & .04 & .00 & .04 & .01 & .04 & -.01 & .03 & -.02 & .04 \\
\hline Mental hyperactivity (Hy2) & .06 & .05 & $.13 * * *$ & .04 & $.11 * * *$ & .04 & $.13 * * *$ & .03 & $.12 * * *$ & .04 \\
\hline Physical health & $-.07 *$ & .08 & .03 & .07 & -.03 & .07 & -.04 & .05 & -.04 & .04 \\
\hline Mental health & $.07 *$ & .08 & .04 & .07 & .06 & .07 & $.08 *$ & .05 & $.07 *$ & .04 \\
\hline Gender $($ male $=0)$ & -.01 & .13 & -.03 & .12 & -.03 & .12 & -.03 & .09 & -.02 & .04 \\
\hline Age (years) & -.01 & .01 & $-.07^{*}$ & .01 & .00 & .01 & -.04 & .01 & -.03 & .04 \\
\hline Education level & .04 & .05 & $.07 *$ & .04 & .05 & .04 & $.07 *$ & .03 & .06 & .04 \\
\hline Firm size & -.06 & .11 & -.01 & .09 & $.08 * *$ & .09 & .00 & .07 & -.01 & .04 \\
\hline$R^{2}$ & .01 & & .04 & & .02 & & .03 & & & \\
\hline
\end{tabular}

$N=788$

Note $* p<.10, * * p<.05, * * * p<.01 . \beta=$ standardized beta

\section{References}

Abbasianchavari A, Moritz A (2020) The impact of role models on entrepreneurial intentions and behavior: a review of the literature. Manag Rev Q. https://doi.org/10.1007/s11301-019-00179-0

American Psychiatric Association (2013) Diagnostic and statistical manual of mental disorders, 5th edn. Author, Arlington

Antshel KM (2018) Attention deficit/hyperactivity disorder (ADHD) and entrepreneurship. Acad Manag Perspect 32(2):243-265

Block J, Kuckertz A (2018) Seven principles of effective replication studies: strengthening the evidence base of management research. Manag Rev Q 68:355-359

Bosma N, Hill S, Ionescu-Somers A, Kelley D, Levie J, Tarnawa A (2020) Global entrepreneurship monitor 2019/2020 global report. Global Entrepreneurship Research Association, London Business School

Brüne N, Lutz E (2020) The effect of entrepreneurship education in schools on entrepreneurial outcomes: a systematic review. Manage Rev Q 70(2):275-305

Canits I, Bernoster I, Mukerjee J, Bonnet J, Rizzo U, Rosique-Blasco M (2019) Attention-deficit/hyperactivity disorder (ADHD) symptoms and academic entrepreneurial preference: is there an association? Small Bus Econ 53(2):369-380

Covin J, Slevin D (1988) The influence of organization structure on the utility of an entrepreneurial top management style. J Manage Stud 25(3):217-234

Csikszentmihalyi M, Seligman ME (2000) Positive psychology: an introduction. Am Psychol 55(1):5-14

Davis JM, Takahashi T, Shinoda H, Gregg N (2012) Cross-cultural comparison of ADHD symptoms among Japanese and US university students. Int J Psychol 47(3):203-210

Dimic N, Orlov V (2014) Entrepreneurial tendencies among people with ADHD. Int Rev Entrep 13(3):187-204

Galvez JF, Thommi S, Ghaemi SN (2011) Positive aspects of mental illness: a review in bipolar disorder. J Affect Disord 128(3):185-190

Hatak I, Chang M, Harms R, Wiklund J (2021) ADHD symptoms, entrepreneurial passion, and entrepreneurial performance. Small Bus Econ. Forthcoming

Hesse M (2013) The ASRS-6 has two latent factors: attention deficit and hyperactivity. J Atten Disord 17(3):203-207

Ingram S, Hechtman L, Morgenstern G (1999) Outcome issues in ADHD: adolescent and adult long-term outcome. Mental retardation and developmental disabilities research reviews 5(3):243-250 
Iwaszuk WM, Weyandt LL, Schepman S, Fouts H, Graff Y, Hays B (1997) Development of the internal restlessness scale. In: Annual meeting of the Western Psychological Association, Seattle, WA

Jamison KR (2005) Exuberance: The passion for life. Vintage, New York

Kessler RC, Adler L, Ames M, Demler O, Faraone S, Hiripi E, Walters EE (2005) The World Health Organization adult ADHD self-report scale (ASRS): a short screening scale for use in the general population. Psychol Med 35(2):245-256

Kessler RC, Adler LA, Gruber MJ, Sarawate CA, Spencer T, Van Brunt DL (2007) Validity of the World Health Organization adult ADHD self-report scale (ASRS) screener in a representative sample of health plan members. Int J Methods Psychiatric Res 16(2):52-65

Kreiser PM, Marino LD, Dickson P, Weaver KM (2010) Cultural influences on entrepreneurial orientation: the impact of national culture on risk taking and proactiveness in SMEs. Entrep Theory Pract 34(5):959-984

Lee SM, Peterson SJ (2000) Culture, entrepreneurial orientation, and global competitiveness. J World Bus 35(4):401-416

Lerner DA, Verheul I, Thurik R (2019) Entrepreneurship and attention deficit/hyperactivity disorder: a large-scale study involving the clinical condition of ADHD. Small Bus Econ 53(2):381-392

Lesch KP (2018) 'Shine bright like a diamond!': is research on high functioning ADHD at last entering the mainstream? J Child Psychol Psychiatry 59(3):191-192

Miller D (1983) The correlates of entrepreneurship in three types of firms. Manag Sci 29(7):770-791

Neumann T (2020) The impact of entrepreneurship on economic, social and environmental welfare and its determinants: a systematic review. Manag Rev Q. https://doi.org/10.1007/s11301-020-00193-7

Nolzen N (2018) The concept of psychological capital: a comprehensive review. Manag Rev Q 68(3):237-277

Okamuro H, Van Stel A, Verheul I (2017) Understanding the drivers of an 'entrepreneurial' economy: lessons from Japan and the Netherlands. In: Bonnet J, Dejardin M, García PLD, Edward Elgar Publishing (eds) Exploring the entrepreneurial society. Edward Elgar Publishing Limited, Northampton, MA

Polanczyk G, De Lima MS, Horta BL, Biederman J, Rohde LA (2007) The worldwide prevalence of ADHD: a systematic review and metaregression analysis. Am J Psychiatry 164(6):942-948

Suzuki KI, Kim SH, Bae ZT (2002) Entrepreneurship in Japan and Silicon Valley: a comparative study. Technovation 22(10):595-606

Verheul I, Block J, Burmeister-Lamp K, Thurik R, Tiemeier H, Turturea R (2015) ADHD-like behavior and entrepreneurial intentions. Small Bus Econ 45(1):85-101

Verheul I, Rietdijk W, Block J, Franken I, Larsson H, Thurik R (2016) The association between attentiondeficit/hyperactivity (ADHD) symptoms and self-employment. Eur J Epidemiol 31(8):793-801

Weyandt LL, Iwaszuk W, Fulton K, Ollerton M, Beatty N, Fouts H, Schepman S, Greenlaw C (2003) The internal restlessness scale: performance of college students with and without ADHD. J Learn Disabil 36(4):382-389

Wiklund J, Hatak I, Patzelt H, Shepherd DA (2018) Mental disorders in the entrepreneurship context: when being different can be an advantage. Acad Manag Perspect 32(2):182-206

Wismans A, Thurik R, Verheul I, Torrès O, Kamei K (2020) Attention-deficit hyperactivity disorder symptoms and entrepreneurial orientation: a replication note. Appl Psychol 69(3):1093-1112. https ://doi.org/10.1111/apps.12247

Yu W, Wiklund J, Pérez-Luño A (2019) ADHD symptoms, entrepreneurial orientation (EO), and firm performance. EntrepTheory Pract. https://doi.org/10.1177/1042258719892987

Publisher's Note Springer Nature remains neutral with regard to jurisdictional claims in published maps and institutional affiliations. 


\section{Affiliations}

\section{Annelot Wismans $^{1,2} \mathbb{D} \cdot$ Katsuyuki Kamei $^{4} \cdot$ Roy Thurik $^{1,2,3} \cdot$ Olivier Torrès $^{3,5}$}

Katsuyuki Kamei

kamei@kansaisu.ac.jp

Roy Thurik

thurik@ese.eur.nl

Olivier Torrès

olivier.torres@umontpellier.fr

1 Department of Applied Economics, Erasmus School of Economics, Erasmus University Rotterdam, PO Box 1738, 3000 DR Rotterdam, The Netherlands

2 Erasmus University Rotterdam Institute for Behavior and Biology, Erasmus University Rotterdam, PO Box 1738, 3000 DR Rotterdam, The Netherlands

3 Montpellier Business School, 34185 Montpellier Cedex 4, France

4 Faculty of Societal Safety Sciences, Kansai University, Hakubaicho 7-1 Takatsuki, Osaka 5691098, Japan

5 Institute Montpellier Management, University of Montpellier, Espace Richter 34960 Cedex, 208 Rue Vendémiaire, 34000 Montpellier, France 